\title{
Calcio en orina de primera micción como predictor de preeclampsia
}

\author{
Rodrigo Palma B. ${ }^{* *}$; Jairo de la Cruz S. ${ }^{2 * *}$
}

\begin{abstract}
RESUMEN: Deseamos obtener una prueba de diagnóstico predictivo temprano para preeclampsia, mediante la detección de los niveles urinarios de calcio en muestras simples, ya que en varios estudios se ha visto que en pacientes con preeclampsia la excreción urinaria de calcio se encuentra disminuida.
\end{abstract}

Se seleccionaron 70 pacientes de la consulta de control prenatal luego de la semana 14 de gestación en el Hospital Militar Central.

El $31.4 \%$ de las pacientes estudiadas presentó preeclampsia, el promedio de calcio urinario en las pacientes que presentaron preeclampsia fue de $34.6 \mathrm{mg} / \mathrm{l}$; el promedio en las que no presentaron preeclampsia fue de $35.8 \mathrm{mg} / \mathrm{l}$ (p 0.081 ), al correlacionar los resultados entre primigestantes y multigestantes, se encontró diferencia estadísticamente significativa entre los niveles de excreción de calcio urinario de ambos grupos.

PALABRAS CLAVES: Preeclampsia, calcio, predictor.

SUMMARY: We want to get an early predictive diagnostic proof for preeclampsia, by the detection of the urine levels of calcium in simple samples; since some studies have shown that patients with preeclampsia, have the calcium urinary excretions in lower levels.

Seventy patients have been selected from the prenatal control office hours after the 14th. Week of gestation in the Military Central Hospital.

The $31.4 \%$ of the patients that were studied presented preeclampsia, the average of urine calcium in the patients that presented preeclampsia was the $34.6 \mathrm{mg} / \mathrm{l}$, the average in those patients that didnt present preeclampsia was $35.8 \mathrm{mg} / \mathrm{l}(\mathrm{p} 0.081$ ) when the results between women with first pregnancy and multiparous were related, it was found a estadistic significative difference between the calcium urinarium excretion of both groups.

KEY WORDS: Preeclampsia, calcium, prediction.

Setenta pacientes con embarazos de segundo trimestre, fueron tomadas para evaluación de los niveles de calcio en muestra simple de orina de primera micción. Después del parto, se revisaron las $\mathrm{HC}$ para determinar si habían presentado preeclampsia. 22 pacientes $(31,4 \%)$ presentaron preeclampsia durante la gestación. El promedio del calcio urinario en las pacientes que posteriormente presentaron preeclampsia fue de 34,6 meq $\backslash 1 \mathrm{y}$ el promedio de las que no presentaron preeclampsia fue de $35,8 \mathrm{meq} \backslash 1(\mathrm{P}>0,05)$, no existiendo diferencia estadísticamente significativa.

Al correlacionar los niveles de calcio con la paridad, se encontró un promedio de 21,7 meq $\backslash 1$ en primigrávidas y de 38,3 meq $\backslash 1$ en multigestantes (P 0.0103), lo cual es estadísticamente significativo.

Se concluye que existe una diferencia significativa en la excreción urinaria de calcio entre primigestantes y multigestantes, lo que hace necesario que se realicen

Residente segundo año Ginecología y Obstetricia. Hospital Militar Central.

Jefe Sección Ginecología y Obstetricia. Hospital Militar Central. trabajos con grupos de primigestantes y otros trabajos con grupos de multigestantes, para correlacionar de una manera más objetiva la excreción urinaria de calcio y el posterior desarrollo de preeclampsia.

La Hipertensión complica por lo menos el $10 \%$ de todos los embarazos (24\%). Los medios de diagnóstico predictivo temprano para preeclampsia más usados son: Presión arterial media, Roll over test, test de la Angiotensina, proteinuria y creatinuria $(1,3-5,7-8,11-$ 12,14-16,21-23,25-27). Uno de los métodos predictivos propuesto recientemente, ha sido la determinación del calcio en orina, ya que según los estudios de Taufield (24) se determinó que el calcio en orina de pacientes preeclámpticas se encontraba disminuido. Existiendo la posibilidad de que esta disminución en el calcio urinario suceda antes de que se presente la preeclampsia- se han realizado estudios en orina de 24 horas y de la relación calcio-creatinuria en muestras seriadas, para determinar el valor predictivo del calcio en orina (18-20).

Para simplificar el método de calcio en orina de 24 horas, se realizó un trabajo de concordancia diagnóstica entre niveles de calcio en muestras de orina de primera 
micción tomadas a partir del segundo trimestre y el resultado final de la gestación en cuanto a si se presentó o no preeclampsia.

\section{Materiales y métodos}

Setenta mujeres embarazadas normotensas, luego de la semana 14 de gestación, que acudieron a consulta de control prenatal en el Hospital Militar Central, participaron en el estudio. Se excluyeron pacientes con historia de enfermedad renal, diabetes, proteinuria, HTA crónica o alguna otra enfermedad crónica. De las 70 pacientes incluidas en el estudio, 12 eran primigestantes y las otras 58 multigestantes.

Se recolectó una muestra de orina de primera micción a cada paciente, a la cual se le determinaron los niveles de Calcio con técnica de espectofotómetro de llama. Al final de la gestación se analizaron las Historias Clínicas para determinar cuales presentaron preeclampsia, y se aplicó la prueba estadística de Monn-Whitney para probar diferencias de medias y determinar si hay diferencia estadísticamente significativa y hallar la sensibilidad, especificidad y valores predictivos.

\section{Resultados}

La tabla 1 muestra la distribución según paridad. De las 70 pacientes $12(17,1 \%)$ eran primigestantes.

Veintidós de las pacientes $(31,4 \%)$ incluidas en el estudio, presentaron preeclampsia. tabla 2 .

El promedio de calcio urinario en las pacientes que presentaron preeclampsia fue de $34,6 \mathrm{Meq} \backslash 1$; y el prome-

Tabla 1

PARIDAD

\begin{tabular}{|c|c|c|c|c|c|}
\hline \multirow[t]{2}{*}{ Value Label } & $\begin{array}{c}\text { Value } \\
1.0 \\
2.0\end{array}$ & $\begin{array}{c}\text { Frequency } \\
12 \\
58\end{array}$ & $\begin{array}{c}\text { Percent } \\
17.1 \\
82.9\end{array}$ & $\begin{array}{c}\text { Valid } \\
\text { Percent } \\
17.1 \\
82.9\end{array}$ & $\begin{array}{c}\text { Cum } \\
\text { Percent } \\
17.1 \\
100.0\end{array}$ \\
\hline & Total & 70 & 100.0 & 100.0 & \\
\hline
\end{tabular}

Tabla 2

RESUL

\begin{tabular}{|c|c|c|c|c|c|}
\hline \multirow[t]{2}{*}{ Value Label } & $\begin{array}{c}\text { Value } \\
1.0 \\
2.0\end{array}$ & $\begin{array}{l}\text { Frequency } \\
\qquad \begin{array}{c}22 \\
48\end{array}\end{array}$ & $\begin{array}{c}\text { Percent } \\
31.4 \\
68.6\end{array}$ & $\begin{array}{c}\text { Valid } \\
\text { Percent } \\
31.4 \\
68.6\end{array}$ & $\begin{array}{c}\text { Cum } \\
\text { Percent } \\
31.4 \\
100.0\end{array}$ \\
\hline & Total & 70 & 100.0 & 100.0 & \\
\hline
\end{tabular}

dio de las que no presentaron preeclampsia fue de 35,8 Meq\1 (P 0.081), no encontrándose diferencia estadísticamente significativa. tabla 3 .

Se correlacionó el promedio de calcio urinario entre primigestantes y multigestantes (tabla 4) encontrándose una P 0.0103 lo cual es estadísticamente significativo.

\section{Conclusiones}

En el presente estudio no se encontraron diferencias en los niveles de calcio urinario entre las pacientes que presentaron preeclampsia con respecto a las que no presentaron preeclampsia; sin embargo, se demostró una diferencia significativa en los niveles de calcio urinario en pacientes primigestantes con relación a las
Tabla 3

\section{MEQ/LI \\ BY RESUL}

\begin{tabular}{|c|c|c|}
\hline Mean Rank & Cases & \\
\hline 34.66 & 22 & Resul $=1.0$ \\
\hline \multirow[t]{3}{*}{35.89} & 48 & Resul $=2.0$ \\
\hline & 70 Total & \\
\hline & & Corrected for Ties \\
\hline $\mathrm{U}$ & W & 2-tailed P \\
\hline 509.5 & 762.5 & -.2341 .8149 \\
\hline
\end{tabular}


multigestantes, lo que hace suponer que la excreción urinaria de calcio al estar modificada de acuerdo a la paridad, debe ser valorada en grupos separados de primigestantes y multigestantes para poder tener un resultado más objetivo de la relación predictiva del calcio urinario y el desarrollo de preeclampsia, tal como lo han sugerido anteriores publicaciones (18-20), pero que en este estudio no fue confirmado.

\section{BIBLIOGRAFIA}

1. Arias F. High risk pregnancy and delivery: 1984; $91: 120$.

2. Beliza J et al. The relationship between calcium intake and pregnancy induced hypertension. Am. J. Obs. and Gyn. 1989; 158 (4): 898-902.

3. Borella $\mathrm{P}$ et al. Maternal plasma concentrations of magnesium. calcium, zinc and cupper en normal and pathological pregnancy. Sci total Env. 1990; 99: 67-76.

4. Chesley, LC. Clinical significance of elevated mean arterial pressure in the second trimester. Am. J. Obs. Gyn. 1988; 159 (2): 275-279.

5. Dekker GA et al. Hypocalciuria of preeclampsia. Am. J. Obs. Gyn. 1991; 165 (1): 160-172.

6. Frenkel $\mathrm{Y}$ et al. Early detection of preeclampsia is independent of parathyroid hormone level. Obs. Gyn. 1991; 75 (5): 689-691.

7. Hernández C. Eclampsia. Clin. Obs. Gyn. 1990; 33 (3): 460-466.

8. Hume RF et al. Fetal umbilical artery doppler response to graded marternal aerobic exercise. Am. J. Obs. Gyn. 1990; 163 (3) 826-829.

9. Jubiz $\mathrm{HA}$ al. Morbimortalidad materna y perinatal en pacientes con preeclampsia y eclampsia. Rev. Col. Obs. y Gin. 1980; 31 (5): 294 303.

10. Kilby MD et al. A cross sectional study of basal platelet intracelular free calcium concentration in normotensive and hypertensive primigravid patients. Clin. Sci. 1990; 78 (1): 75-80.

11. Lindheimer MD et al. Preeclampsia: pathophysiology, diagnosis and management. Ann. Rev. Med. 1989; 40: 233-250.

12. Lindheimer MD. Diagnosis and management of hypertension complicating pregnancy. Am. J. Kidney Dis. 1989; 13 (6): 17-27.

13. Lopez $P$ et al. Calcium supplementation reduces the risk of pregnancy hypertension in an Andes population. Br. J. Obs. Gyn. 1989; 96: 648655.

14. Mc Carron D. The calcium deficiency. Hipotesis of Hypertension. Ann. In. Med. 1987; 107 (6): 919-922.

15. 0'Brieb MD. Predicting Preeclampsia. Obs. Gyn. 1990; 75 (3): 445 452.

16. Remuzzi $G$ et al. Prevention and treatment of pregnancy associated hypertension. Am. J. Kidney Dis. 1991; 18 (3): 285-305.

17. Richardson D. Et. 11. Diagnostic test in obstetrics a method of improved evaluation. Am. J. Obs. Gyn. 1990; 152 (3): 445-452.
18. Rodríguez $\mathrm{MH}$ et al Calcium/Creatinine ratio and microalbuminaria in the prediction of preeclampsia. Am. J. Obs. Gyn. 1988; 159 (6): $1452-1455$.

19. Sánchez Ramos̀ et al. Calcium excretion in preeclampsia. Obs. Gyn. 1991; 77 (4) 510-513.

20. Sánchez Ramos et al. Urinary calcium as an early marker for preeclampsia. Obs. Gyn. 1991; 77 (5): 415-688.

21. Schutuz K et al. Serial renal sonographic changes in preeclampsia. J. Ultr. Med. 1990; 9 (7); 415-418.

22. Sibay BM. Medical disorders including hypertensive diseases. Curr. Op. Obs. Gyn. 1991; 3 (1): 28-40.

23. Sibay BM. Pitfalls in diagnosis and management of preeclampsia. Am. J. Obs. Gyn. 1989; 159 (1): 1-5.

24. Taufield PA et al. Hipocalciuria in preeclampsia. N. Eng. Med. 1987; 316 (12): 715-718.

25. Thorp JM et al. Von willebrand factor multimetric levels and patterns in patients with severe preeclampsia. Obs. Gyn. 1990; 75 (2): 163167.

26. Villar $\mathrm{M}$ et al. Clinical significance of elevated mean arterial pressure in second trimester and thereshold increase in sistolic or diastolic blood presure during third trimester. Am. J. Obs. Gyn. 1989; 160 (2) 419-423.

27. Villar M. Gin. Obs. tem Ac. Eclampsia. 1988; 15 (2); 315-371.

28. Walker JJ et al. Can platelet volume predict progressive hypertensive disease in pregnancy. Am. J. Obs. Gyn. 1989; 161 (3): 676-679.

29. Zemel $\mathrm{MB}$ et al. Altered platetlet calcium metabolism as an early predictor of increased peripheral vascular resistance and preeclampsia in urban black women. N. Eng. J. Med. 1990; 323 (7): 434-438.

30. Landazábal A. Calcio en orina de 24 horas para diagnóstico de hipertensión inducida por el embarazo. Trab Asc niv III Hosp Mil Cent. 1991. 